\title{
Thermodynamic Modeling of Ternary Liquid-Liquid Systems with Forming Immiscibility Islands
}

\author{
Andre Zuber ${ }^{2}$, Rodrigo Raimundo ${ }^{1}$, Marcos Rogério Mafra ${ }^{1}$, Lúcio Cardozo Filho ${ }^{2}$, José \\ Vladimir Oliveira ${ }^{3}$ and Marcos Lúcio Corazza ${ }^{*}$ \\ ${ }^{I}$ Departamento de Engenharia Química; Universidade Federal do Paraná; Curitiba - PR - Brasil. ${ }^{2}$ Departamento \\ de Engenharia Química; Universidade Estadual de Maringá; Maringá - PR - Brasil. ${ }^{3}$ Departamento de Engenharia \\ Química e de Alimentos; Universidade Federal de Santa Catarina; Florianópolis - SC - Brasil
}

\begin{abstract}
The aim of this work was to study the application of a stochastic algorithm to correlate the experimental data of island-type systems in ternary systems. Thermodynamic NRTL and UNIQUAC models were used to evaluate the activity coefficients. Results regarding the application of the stochastic algorithm were in good agreement with those presented in the current literature. However, generalization of the method proposed in the present work remained an intriguing and complex task so as to reach broad conclusions.
\end{abstract}

Key words: LLE, island-type, stochastic algorithm, NRTL, UNIQUAC

\section{INTRODUCTION}

Ternary liquid mixtures that present closed miscibility gaps are classified as Type 0 systems, according to the classification proposed by Treybal (1963) and mentioned by Olaya et al. (2008). At constant pressure and temperature, a liquid-liquid immiscible island is formed when a heterogeneous region of two immiscible liquids (or partially miscible) is completely enclosed by a homogenous liquid region. Thus, there is complete miscibility in all binary pairs of the system involved. The binodal curve of island presents two plait points that limit the tie-lines in both sides of the island (Treybal 1963; Olaya et al. 2008). Liquid-liquid immiscible islands are commonly found in the systems formed by weak acids and bases that reversibly produce a soluble salt, which presents a limited miscibility (Olaya et al. 2008).

Despite of the availability of published experimental data of ternary LLE systems with immiscible islands, thermodynamic modeling of these systems has not been commonly studied (Sørensen et al. 1979a; 1979b; 1980). In fact, just a few studies can be found concerning the application of conventional activity coefficients models for the correlation of such systems. Olaya et al. (2008) reported that the local composition models such as NRTL (Renon and Prausnitz 1968) and UNIQUAC (Abrams and Prausnitz 1975) showed difficulty to predict a ternary LLE region of three completely miscible binary systems. As shown in DECHEMA Chemistry Data Series (Sorensen and Art 1980), the experimental data of Type 0 systems could not be correlated and the binary interaction parameters of thermodynamic models, such as NRTL and UNIQUAC, were thus not available, unlike the situation verified for all other LLE systems (Type I and II systems).

There are only reports on this subject. For example, Olaya et al. (2008) reported the correlation of Type 0 systems (islands occurrence)

\footnotetext{
*Author for correspondence: corazza@ufpr.br
} 
proposing an empirical rule for the parameters of NRTL model to be used in the estimation procedure. The experimental data of Type 0 systems were correlated with the NRTL model using topological concepts related to the Gibbs stability test. From the generalized topological analysis for the NRTL model, it was observed that the sum of the binary parameters for a one of the systems must be negative (characterization of the dissimilar binary pair) while the sum of binary parameters for the other two systems must be positive, i.e.:

$$
\begin{aligned}
& A_{12}+A_{21}<0 \text { (dissimilar binary pair) } \\
& A_{13}+A_{31}>0 \\
& A_{23}+A_{23}>0
\end{aligned}
$$

Equation 1 was the proposition (condition) obtained by the observations on the Gibbs energy surfaces considering the islands formation for various ternary systems. From this, the authors argue that changes must be made in the algorithms for calculation and correlation of LLE in order to correlate efficiently the occurrence of immiscible islands. Another aspect that must be observed in the work of Olaya et al. (2008) was that the optimization algorithm used to fit the experimental data was a modified Simplex method (Flexible Simplex method), which was a variant of the Nelder-Mead method with flexible polyhedron (Press et al. 1992). This algorithm is dependent on the heuristic initial parameters estimation, despite the fact that the restriction imposed by the Equation (1) must be fulfilled in the optimization problem formulation to ensure that the final solution found corresponded to the ternary system with the formation of the immiscible island (Olaya et al. 2008). Most currently used algorithms, including simulators and commercial correlation packages for the thermodynamic properties calculations, do not calculate the formation of ternary immiscible islands because to carry out calculations at least one pair of immiscible liquidliquid is required.

Modeling liquid phases in equilibrium with activity coefficient models, such as NRTL and UNIQUAC, frequently provides several local minima, thus making the parameter estimation step a nontrivial issue. In order to reduce the complexity involved, a global optimization algorithm may be needed. For this purpose, some stochastic optimization algorithms have been proposed as interesting alternatives instead of derivative methods (Stragevitch and d'Ávila 1997; Corazza et al. 2008; Ferrari et al. 2009). In a general way, such class of algorithms does not require some properties of the objective function needed to find the global solution as required by the deterministic techniques.

This work aimed to study the application of a stochastic algorithm for LLE modeling with NRTL and UNIQUAC models to correlate the experimental data of ternary systems showing LL immiscible islands (or the tendency of formation). For this purpose, the stochastic Simulated Annealing algorithm presented by Ferrari et al. (2009) was employed to estimate the binary interaction parameters of the thermodynamic models. The split calculations (LLE tie lines) were performed from the results of the phase stability test to ensure the reliability of LLE solutions obtained for each group of parameters obtained from the stochastic optimization algorithm. The main goal was to discuss the LLE island type ternary systems, bringing insights and improving the interpretations on the basis of the so-called molecular thermodynamics regarding phase behavior and physical meaning of adjusted parameters obtained from data correlation. Specifically, the following systems were investigated: water + DMSO + THF at $293.15 \mathrm{~K}$ and water + acetone + phenol at $323.15 \mathrm{~K}, 333.15$ $\mathrm{K}, 353.15 \mathrm{~K}, 393.15 \mathrm{~K}, 413.15 \mathrm{~K}, 433.15 \mathrm{~K}$ and $453.15 \mathrm{~K}$.

\section{ALGORITHMS}

For the LLE calculations and parameter estimation of thermodynamic models, the algorithm presented by Ferrari et al. (2009) was used. In this algorithm, the LLE calculations are performed based on the phase stability test by analyzing the Gibbs surface tangent plane distance, and once instability is observed, the liquid-liquid equilibrium calculation is performed by applying the multiphase liquidliquid flash algorithm (Michelsen 1980). After calculating the LLE, one phase is tested again, if instability is again observed, i.e., another possible phase in equilibrium (LLLE) is detected; the penalty function is activated. In this work the barrier method in the objective function was used. This strategy avoids the inconsistent set of parameters on the phase number. The same procedure of the objective function penalty is applied if the stability test identifies, for a given tie 
line, a homogeneous region (single phase). Thus, the problem of parameters estimation of NRTL and UNIQUAC models can be formulated as:

$$
\min F O=\sum_{k=1}^{N P} \sum_{j=1}^{n f} \sum_{i=1}^{n c} \frac{\left(x_{i j k}^{c a l c}-x_{i j k}^{e x p}\right)^{2}}{\sigma_{\mathrm{j}}^{2}}+\mathrm{Q} \sum_{\mathrm{k}=1}^{N P} \mathrm{t}_{\mathrm{k}}
$$

where, $x_{i j k}$ are the mole fractions of component $i$ in phase $j$ for the tie line $k, N P$ is the total number of tie lines, $n f$ is the total number of phases, $n c$ is the number of compounds in the system, $\sigma_{j}^{2}$ is the variance of the experimental phase, the parameter $t_{k}$ is the penalty term (in this work, it is used the flash convergence degree) and $\mathrm{Q}$ is the constant term of the penalty $\left(1 \times 10^{6}\right)$.

For the minimization of the objective function (Eq. 2), the Simulated Annealing algorithm was used as also described by Ferrari et al. (2009) without further modifications. To evaluate the fitting quality of the models, the root mean square deviation $(R M S D)$ was used as follows:

$$
100 R M S D=100 \sqrt{\frac{1}{N P \times n f \times n c} \sum_{k=1}^{N P} \sum_{j=1}^{n f} \sum_{i=1}^{n c}\left(x_{i j k}^{\text {exp }}-x_{i j k}^{c a l c}\right)^{2}}
$$

where, $x_{i j k}^{\text {calc }}$ and $x_{i j k}^{e x p}$ referred to calculated and experimental mole fraction, respectively, of component $i$ in phase $j$ for the other point in tie line $k ; N P$ is the total number of tie lines, $n f$ was the total number of phases, $n c$ was the number of compounds in the system.

It must be emphasized that the phase equilibrium calculation algorithm used in this work (experimental data correlation from Type 0 ternary systems) was exactly the same, without any change or adaptation, of that presented and used previously by Ferrari et al. (2009) for the correlation of Type I and II systems.

\section{RESULTS AND DISCUSSION}

Results of data correlation for the same system studied by Olaya et al. (2008) are presented in this work (System 1). Afterwards the results obtained for a system that tended to form the immiscibility island at the temperature considered, but did not occur effectively in the region investigated (System 2) has been reported.

\section{System 1: water(1)+DMSO(2)+THF(3)}

The experimental data for the ternary system water (1) + DMSO(2) + THF(3) was obtained from the LLE database of DECHEMA Chemistry Data Series (Sorensen and Art 1980). Olaya et al. (2008) used this system in their work since the data presented by Wolski (1970) were tie lines. In the present work, two parameters estimations were performed for the NRTL model. In the first case, the values of $\alpha$ were fixed $(\alpha=0.2)$ for the three pairs of the ternary system (Set 1 ), so as to allow the comparison with the procedure presented by Olaya et al. (2008). In the second case, all the parameters (Set 2), $\mathrm{g}_{\mathrm{ij}}$ and $\alpha_{\mathrm{ij}}$, were adjusted. Table 1 presents the parameters fitted for the system water (1) $+\operatorname{DMSO}(2)+\operatorname{THF}(3)$ and Figure 1 shows this ternary system, where the experimental data and results of NRTL model are depicted, using the Set 2 of parameters presented in Table 1. In Table 1, the parameters obtained for the UNIQUAC model are also shown.

Table 1 - Fitted binary interaction parameters of the NRTL and UNIQUAC models for the ternary system

\begin{tabular}{|c|c|c|c|c|}
\hline NRTL & $\mathbf{i}-\mathbf{j}$ & $\Delta g_{i j} / \mathbf{K}$ & $\Delta g_{i i} / \mathbf{K}$ & $\alpha_{i j}$ \\
\hline \multirow[t]{4}{*}{ Set $1^{(*)}$} & $1-2$ & -173.01 & -1031.42 & 0.2 \\
\hline & $1-3$ & 556.89 & 71.45 & 0.2 \\
\hline & $2-3$ & 473.31 & -402.56 & 0.2 \\
\hline & & 100 RMSD & 3.18 & \\
\hline \multirow[t]{3}{*}{$\overline{\text { Set } 2}$} & $1-2$ & -227.67 & -1203.80 & 0.1516 \\
\hline & $1-3$ & 495.06 & 145.86 & 0.2754 \\
\hline & $2-3$ & 636.01 & -383.10 & 0.4531 \\
\hline \multirow[t]{5}{*}{ UNIQUAC $^{(\mathbf{a})}$} & $i-j$ & $\Delta u_{i j} / \mathrm{K}$ & $\Delta u_{i i} / \mathrm{K}$ & \\
\hline & $1-2$ & -380.64 & -848.30 & \\
\hline & $1-3$ & -20.7937 & -204.65 & \\
\hline & $2-3$ & 458.37 & -248.46 & \\
\hline & & 100 RMSD & 3.97 & \\
\hline \multirow[t]{5}{*}{ UNIQUAC $^{(\text {b) }}$} & $i-j$ & $\Delta u_{i j} / \mathrm{K}$ & $\Delta u_{j i} / \mathrm{K}$ & \\
\hline & $1-2$ & -164.65 & -484.91 & \\
\hline & $1-3$ & 116.21 & -7.0767 & \\
\hline & $2-3$ & 149.48 & -145.34 & \\
\hline & & 100 RMSD & 3.40 & \\
\hline
\end{tabular}
water(1) + DMSO(2) + THF(3) at $293.15 \mathrm{~K}$.

This study also tested different values of $\mathrm{R}$ and $\mathrm{Q}$ parameters as shown in Table 1 . In the first case, $R$ and $\mathrm{Q}$ values were obtained from the UNIFACLLE parameters database from Magnussen et al. (1981), and in the second, the values were obtained from the tables of the UNIFAC- 
Dortmund (UNIFAC-D) database from Gmehling et al. (1993).

Figure 1 showed that the LLE modeling results were significantly better than those reported by Olaya et al. (2008). Quantitatively, this was also seen from Table 1 by comparing the root mean square deviation values $(R M S D)$ (Table 1) with $R M S D$ values obtained using the parameters reported by Olaya et al. (2008). The best correlation obtained could be attributed to the optimization algorithm used, which generally afforded a convergence to the global optimum, as demonstrated by Ferrari et al. (2009).

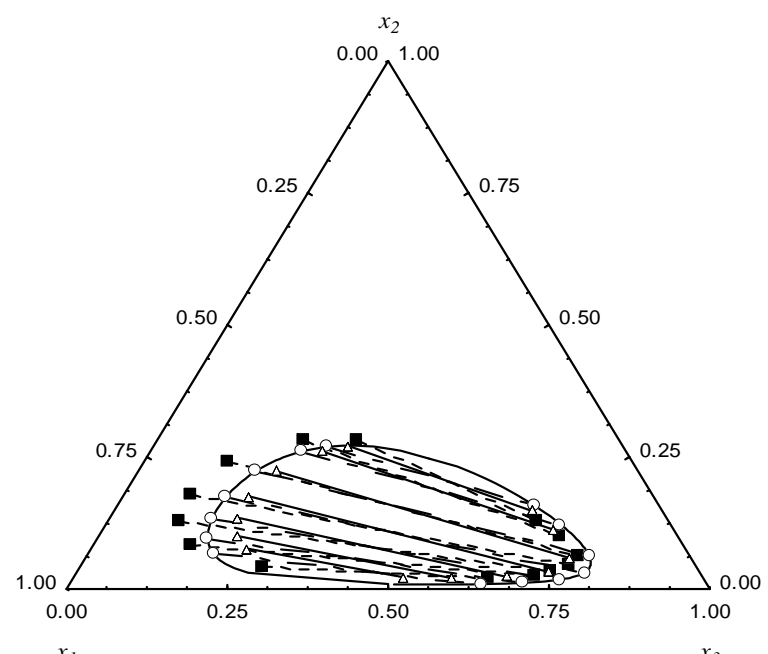

Figure 1 - Comparison of experimental (--) and calculated values using NRTL model ( - , binodal; and $-\mathrm{O}-$, tie lines from this work) for water (1) + DMSO (2) + THF (3) ternary system at $293.15 \mathrm{~K}$ (mole fractions). $(-\leftarrow$, tie lines values calculated using NRTL parameters from Olaya et al. 2008).

Figure 2 shows the result of LLE modeling using the UNIQUAC model with $\mathrm{R}$ and $\mathrm{Q}$ parameters from the Dortmund database (Gmehling et al. 1993). The binary interaction parameters obtained are presented in Table 1.

From the results presented in Table 1 and in Figures 1 and 2, the UNIQUAC model, with R and $\mathrm{Q}$ parameters from the Dortmund database, proved to be more efficient to correlate the LLE data and to predict the formation of liquid-liquid immiscible islands. It also presented a similar ability to correlate this system compared with NRTL model. When R and Q parameter were used from the UNIFAC-LLE databank (Magnussem et al. 1981), the results obtained are slightly poorer when compared to those obtained with the other approach, as shown from the RMSD values in Table 1. In general, both UNIQUAC and NRTL models could be efficiently used to predict the liquid-liquid immiscible islands.

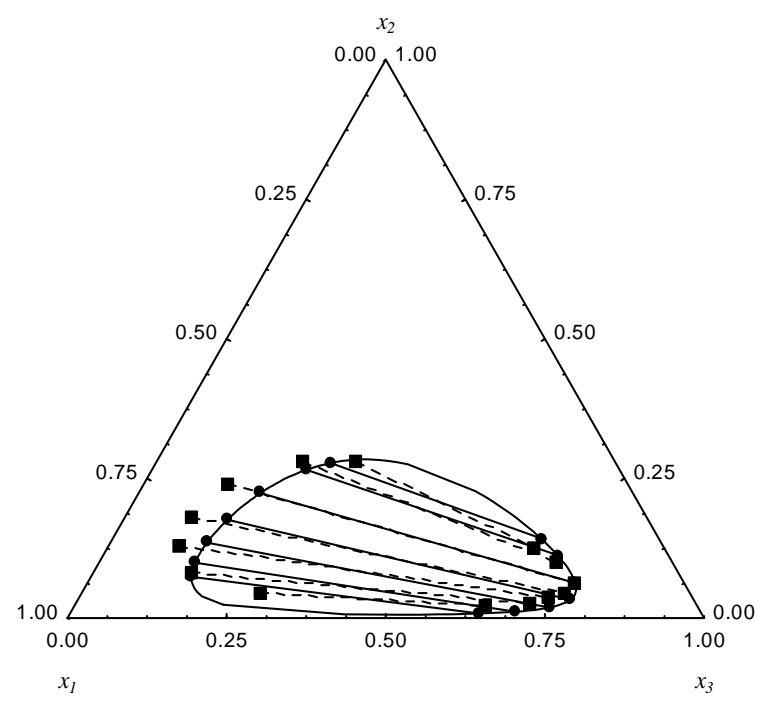

Figure 2 - Comparison of experimental (-- $)$ and calculated values using UNIQUAC model ( - , binodal; and $\rightarrow$ tie lines from this work) for water (1) + DMSO (2) + THF (3) ternary system at $293.15 \mathrm{~K}$ (mole fraction).

However, in the UNIQUAC case, results seemed to be sensitive to the $\mathrm{R}$ and $\mathrm{Q}$ parameters values chosen.

The proposition presented by Olaya et al. (2008), Equation 1, was observed for the parameters obtained in the two sets with the NRTL model (Table 1). The sum of the parameters for the dissimilar pair was $g_{12}+g_{21}<0$, while for the other two pairs the sum was $g_{13}+g_{31}>0$ and $g_{23}+$ $\mathrm{g}_{32}>0$. Olaya et al. (2008) pointed out that for parameter estimation the inequalities (Equation 1) must be incorporated in the optimization algorithm. However, in this work, because of the random search of the stochastic optimization algorithm, the inequality was not introduced into the fitting algorithm. Hence, the empirical rule proposed by Olaya et al. (2008) seemed to be a characteristic of systems where immiscible islands occurred.

Considering that the parameters $\Delta g_{i j}$ for NRTL model (or $\Delta u_{i j}$ for UNIQUAC model) were closely 
related to energy difference between the cross $\left(\lambda_{\mathrm{ij}}=\lambda_{\mathrm{ji}}\right)$ and pure $\left(\lambda_{\mathrm{ii}}\right.$ or $\left.\lambda_{\mathrm{jj}}\right)$ pair intermolecular potential, i.e.,

$$
\Delta \mathrm{g}_{\mathrm{ij}}=-\frac{\left(\lambda_{i j}-\lambda_{i i}\right)}{T}
$$

where $\lambda_{\mathrm{ij}}$ is the pair energy parameter relative to the ideal gas state at the minimum intermolecular potential, thus always a negative parameter (see for example, Prausnitz et al. 1999).

Equation (4) showed that if interactions between the same molecules, intermolecular forces in the pure fluids ii and/or jj are greater (in module, which means more negative values) than those occurring between ij pair, then $\Delta g_{i j}$ and $\Delta \mathrm{g}_{\mathrm{ji}}$ would be negative, and hence immiscibility might occur. Conversely, if the intermolecular forces of similar intermolecular pairs (ii and jj) were lower (in module), or presented the same order of magnitude of dissimilar (cross) interaction pairs, ij, then it would be natural to expect the complete miscibility of the system and in this case $\Delta g_{i j}$ and $\Delta g_{j i}$ would be positive. This was physically sound, as it was expected that more intense the intermolecular forces between the dissimilar pairs (ij) compared to the pure interactions, more homogeneous should tend to be the mixture. This explained the values of the parameters found in this work, presented in Table 1. The fundamental intermolecular potential energy approach evoked here also explained why as the $\mathrm{A}_{12}$ and $\mathrm{A}_{21}$ parameters shown in Figure 3 in the work of Olaya et al. (2008) increased (in module) the immiscibility island (LL) region and why one should expect positive values for the other two pairs (13 and 23) - complete miscibility. For the positive fixed parameters sets of pairs 13 and 23 $\left(\mathrm{A}_{13}=\mathrm{A}_{31}=\mathrm{A}_{23}=\mathrm{A}_{32}=120\right.$ in the work of Olaya et al. 2008) as $A_{12}$ and $A_{21}$, parameters were reduced a Type 1 LLE appeared and the same rational could be applied to expect the appearance of Type 2 and Type 3 LLE systems. Thus, it can be seen that Equation (1) presents no longer strictly empirical relations since that rules came from well-based thermodynamic fundamentals.

\section{System 2: water(1) + acetone $(2)+$ phenol(3)}

This system was experimentally studied by Mafra and Krähenbühl (2006). For the isotherms presented in the literature (323.15 and $333.15 \mathrm{~K}$ ), there was no complete formation of the liquidliquid immiscible island for this system; however, the experimental data showed that there was a strong tendency in the occurrence of islands. For this system at $323.15,333.15,343.15$ and 353.15 $\mathrm{K}$, experimental binodal curves were available in the literature, where immiscibility islands at higher temperatures were observed by Guttman and Wilczura (1997).

In the present study, the NRTL and UNIQUAC models were employed to correlate the tie lines reported in the literature at 323.15 and $333.15 \mathrm{~K}$ (Mafra and Krähenbühl 2006). For the NRTL model, the experimental data were correlated using the fixed value of $\alpha_{\mathrm{ij}}$ (Set 1) and it were also adjusted (Set 2), where a better fitting could be observed considering the Set 2. For the UNIQUAC model, the $\mathrm{R}$ and $\mathrm{Q}$ values of pure components were taken from the Dortmund database (UNIFAC-D) and from the UNIFACLLE database. Table 2 shows the fitted parameters values for both models, as well as the root mean square deviation values (RMSD).

Table 2 - Fitted binary interaction parameters of the NRTL and UNIQUAC models for the ternary system water(1) + acetone (2) + phenol (3) at $323.15 \mathrm{~K}$ and $333.15 \mathrm{~K}$.

\begin{tabular}{|c|c|c|c|c|}
\hline NRTL & $\mathbf{i}-\mathbf{j}$ & $\Delta g_{i j} / \mathbf{K}$ & $\Delta g_{i i} / \mathrm{K}$ & $\alpha_{i j}$ \\
\hline \multirow{4}{*}{ Set $1^{(*)}$} & $1-2$ & 33.08 & 590.03 & 0.2 \\
\hline & $1-3$ & 1723.03 & -512.32 & 0.2 \\
\hline & $2-3$ & -486.06 & -898.09 & 0.2 \\
\hline & & 100 RMSD & 1.66 & \\
\hline \multirow{4}{*}{ Set 2} & $1-2$ & 336.99 & 34.13 & 0.2194 \\
\hline & $1-3$ & 1090.93 & 1241.06 & 0.4694 \\
\hline & $2-3$ & -257.12 & -899.41 & 0.2922 \\
\hline & & 100 RMSD & 1.06 & \\
\hline \multirow[t]{5}{*}{ UNIQUAC $^{(\mathbf{a})}$} & $\mathrm{i}-\mathrm{j}$ & $\Delta u_{i j} / \mathrm{K}$ & $\Delta u_{j i} / \mathrm{K}$ & \\
\hline & $1-2$ & -66.61 & 489.78 & \\
\hline & $1-3$ & 430.16 & -213.65 & \\
\hline & $2-3$ & 4096.41 & -481.13 & \\
\hline & & 100 RMSD & 1.52 & \\
\hline \multirow[t]{5}{*}{ UNIQUAC $^{(\mathbf{b})}$} & $\mathrm{i}-\mathrm{j}$ & $\Delta u_{i j} / \mathrm{K}$ & $\Delta u_{j i} / \mathrm{K}$ & \\
\hline & $1-2$ & -246.70 & 37.15 & \\
\hline & $1-3$ & 583.12 & -201.49 & \\
\hline & $2-3$ & -51.96 & -773.67 & \\
\hline & & 100 RMSD & 1.11 & \\
\hline \multicolumn{5}{|c|}{$\begin{array}{l}\text { (*) fixed } \alpha_{\mathrm{ij}} \text { values; }{ }^{(\mathrm{a})} \mathrm{R} \text { and } \mathrm{Q} \text { values obtained from Magnussen et al. } \\
\text { (1981) and }{ }^{(\mathbf{b})} \text { Gmehling et al. (1993). Fitted parameters were }\end{array}$} \\
\hline & & & & \\
\hline
\end{tabular}

Although the immiscible island was not effectively formed for the system water(1)+acetone (2)+phenol(3) at 323.15 and $333.15 \mathrm{~K}$, Table 2 showed that the parameters obtained for the NRTL model presented the same behavior as proposed by 
Olaya et al. (2008) for the pair 1-3, and the "molecular analysis" discussed above was still valid. For the UNIQUAC model, this proposition did not show any tendency to follow the Equation 1.

Table 3 shows the root mean square deviation and the absolute error values for NRTL and UNIQUAC models fitted in this work for each temperature. This table showed the results when the UNIFAC-D and UNIFAC-LLE models were used and also the $R M S D$ values and absolute error using the interaction parameters for NRTL and UNIQUAC models presented in the literature (Mafra and Krähenbühl 2006).

Table 3 - Values of root mean square deviation $(R M S D)$ and absolute deviation $(A D)$ using different activity coefficient models used in this work and literature for the system water (1) + acetone (2) + phenol (3).

\begin{tabular}{|c|c|c|c|}
\hline Model & T / K & $100 \mathrm{RMSD}$ & $A D(\%)$ \\
\hline \multirow{2}{*}{ UNIFAC-LLE } & 323.15 & 9.26 & 6.82 \\
\hline & 333.15 & 11.53 & 8.47 \\
\hline \multirow{2}{*}{ UNIFAC-D } & 323.15 & 1.76 & 1.15 \\
\hline & 333.15 & 1.26 & 0.69 \\
\hline \multirow{2}{*}{$\begin{array}{l}\text { UNIQUAC (Mafra and } \\
\text { Krähenbühl 2006) }\end{array}$} & 323.15 & 1.21 & 0.67 \\
\hline & 333.15 & 0.97 & 0.52 \\
\hline \multirow{2}{*}{ UNIQUAC (this work) } & 323.15 & 1.17 & 0.78 \\
\hline & 333.15 & 1.04 & 0.64 \\
\hline \multirow{2}{*}{$\begin{array}{l}\text { NRTL (Mafra and } \\
\text { Krähenbühl 2006) } \\
\end{array}$} & 323.15 & 1.24 & 0.70 \\
\hline & 333.15 & 1.02 & 0.58 \\
\hline \multirow{2}{*}{ NRTL (this work) } & 323.15 & 1.13 & 0.74 \\
\hline & 333.15 & 0.98 & 0.61 \\
\hline
\end{tabular}

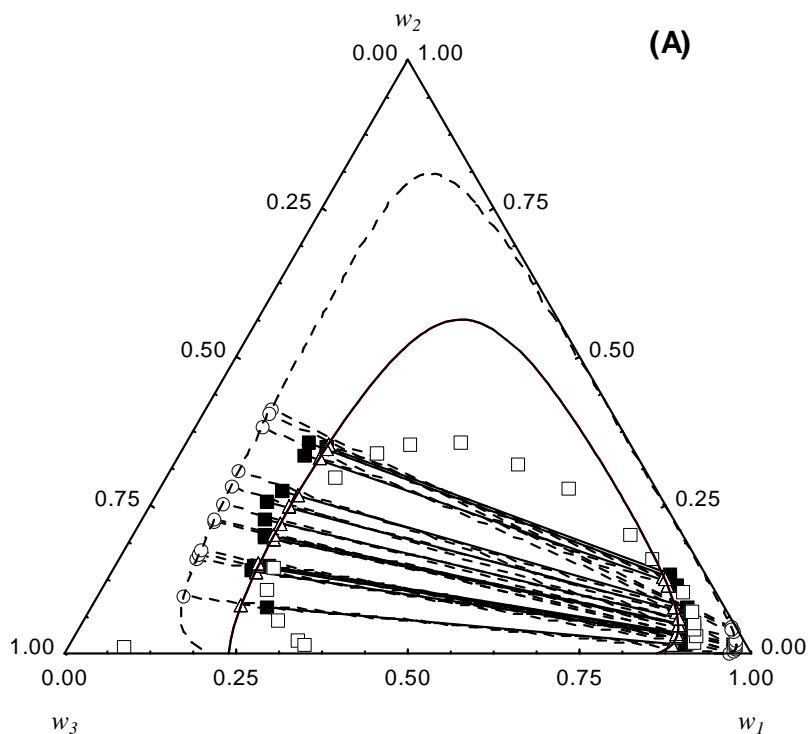

Figures 3(A) and 3(B) show the ternary diagrams for this system with the results obtained from the predictions using the UNIFAC models. Evidently the UNIFAC-D model presented the more satisfactory results for the prediction of the LL immiscible region than the UNIFAC-LLE model. Figures 4(A) and 4(B) show the ternary diagrams for water(1)+acetone (2)+phenol(3) system correlated with NRTL model. The values of tie lines and binodal curves using the parameters for the NRTL model presented in the literature are also shown. It was observed that the correlation presented in this work represented more accurately the tendency to island formation. This was observed at 323.15 and $333.15 \mathrm{~K}$.

For the NRTL model presented in the previous figures, the correlation for the UNIQUAC model presented in this work was better than the fit presented in the literature, as shown in Figures 5(A) and 5(B) for the isotherms of 323.15 and $333.15 \mathrm{~K}$. It should be noted that the values of $\mathrm{R}$ and $\mathrm{Q}$ used in this work were obtained from the Dortmund database (Fig. 5(A) and 5(B)). In general, the algorithm used to correlate LLE data from water(1) + acetone $(2)+\operatorname{phenol}(3)$ system at 323.15 and $333.15 \mathrm{~K}$, presented satisfactory results of $R M S D$, lower than those reported in the literature. Moreover, both the NRTL and UNIQUAC models showed good ability to correlate the data from this LLE system.

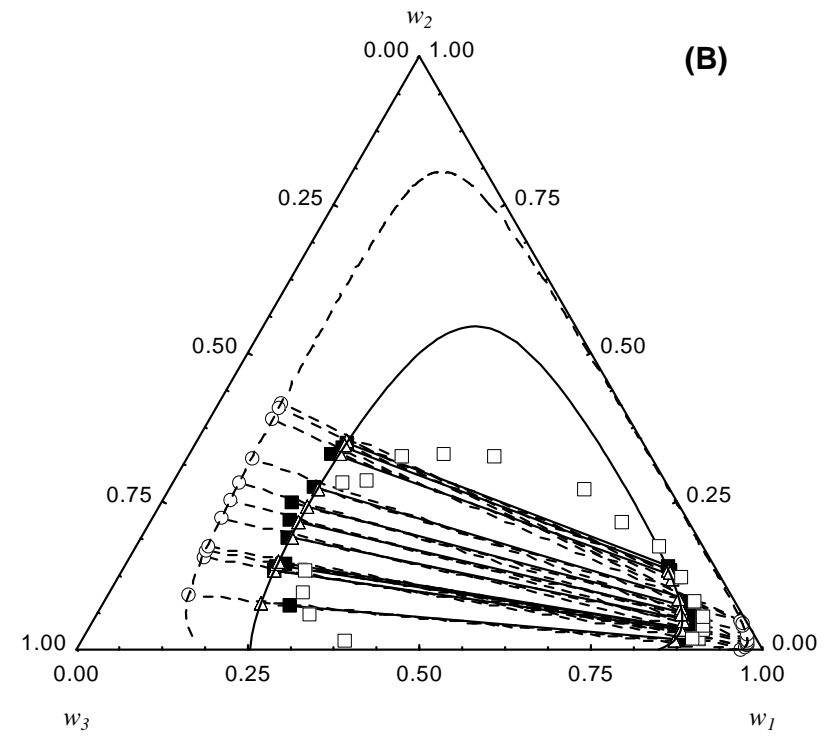

Figure 3 - Comparison of experimental ( $\square$, binodal data by Guttman and Wilczura 1997, $\rightarrow$ tie lines by Mafra and Krähenbühl 2006) and calculated values using UNIFAC-LLE ( -...-, binodal; $--\mathrm{O}^{--}$, tie lines) and UNIFAC-D ( - , binodal; $--^{-\cdot}$, tie lines $)$ models for water (1) + acetone (2) + phenol (3) ternary system (A) $323.15 \mathrm{~K}$ and (B) $333.15 \mathrm{~K}$ (mass fraction). 

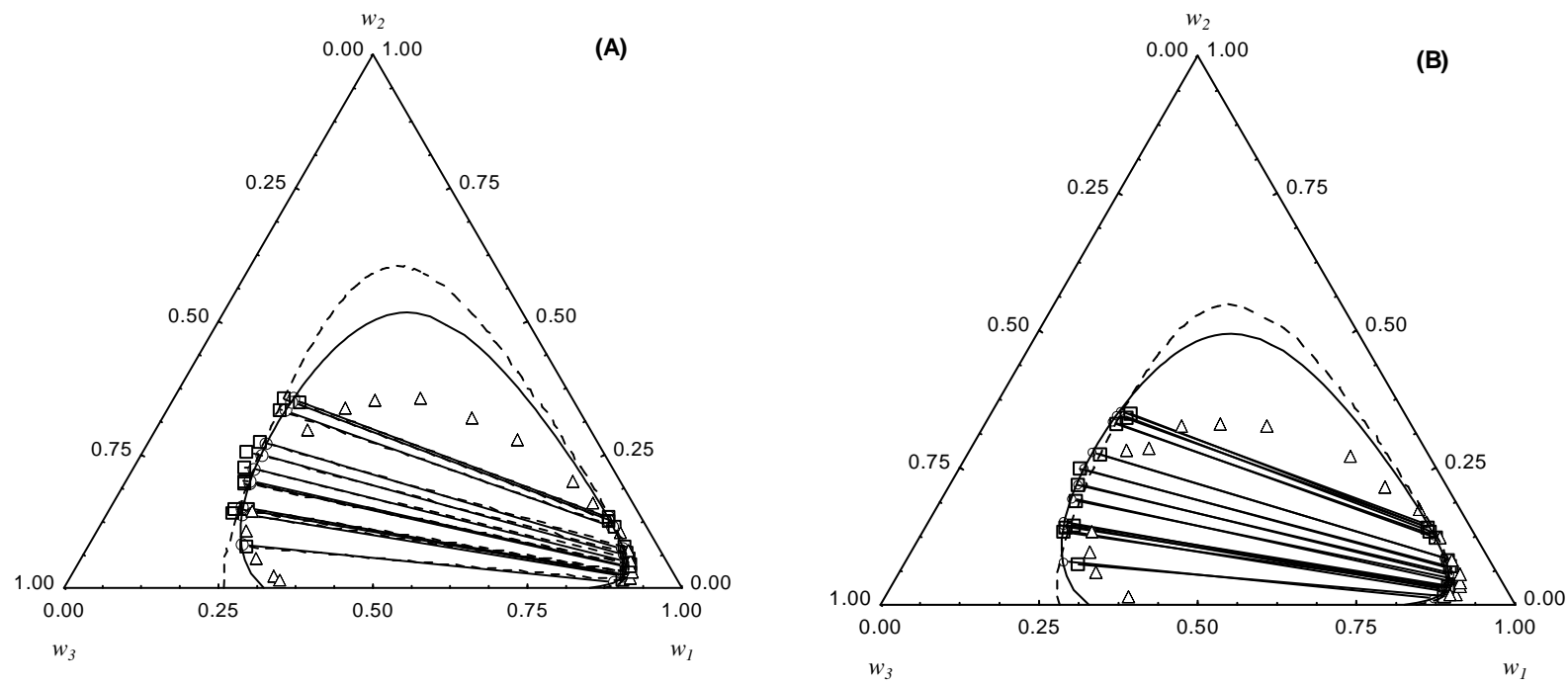

Figure 4 - Comparison of experimental ( $\bullet$, binodal data by Guttman and Wilczura 1997, $\square-$ tie lines by Mafra and Krähenbühl 2006) and calculated values using NRTL model ( $-\mathbf{O}$, this work; -...-., with parameters from Mafra and Krähenbühl 2006) for water(1) + acetone(2) + phenol(3) system (A) $323.15 \mathrm{~K}$ and (B) $333.15 \mathrm{~K}$ (in mass fraction).
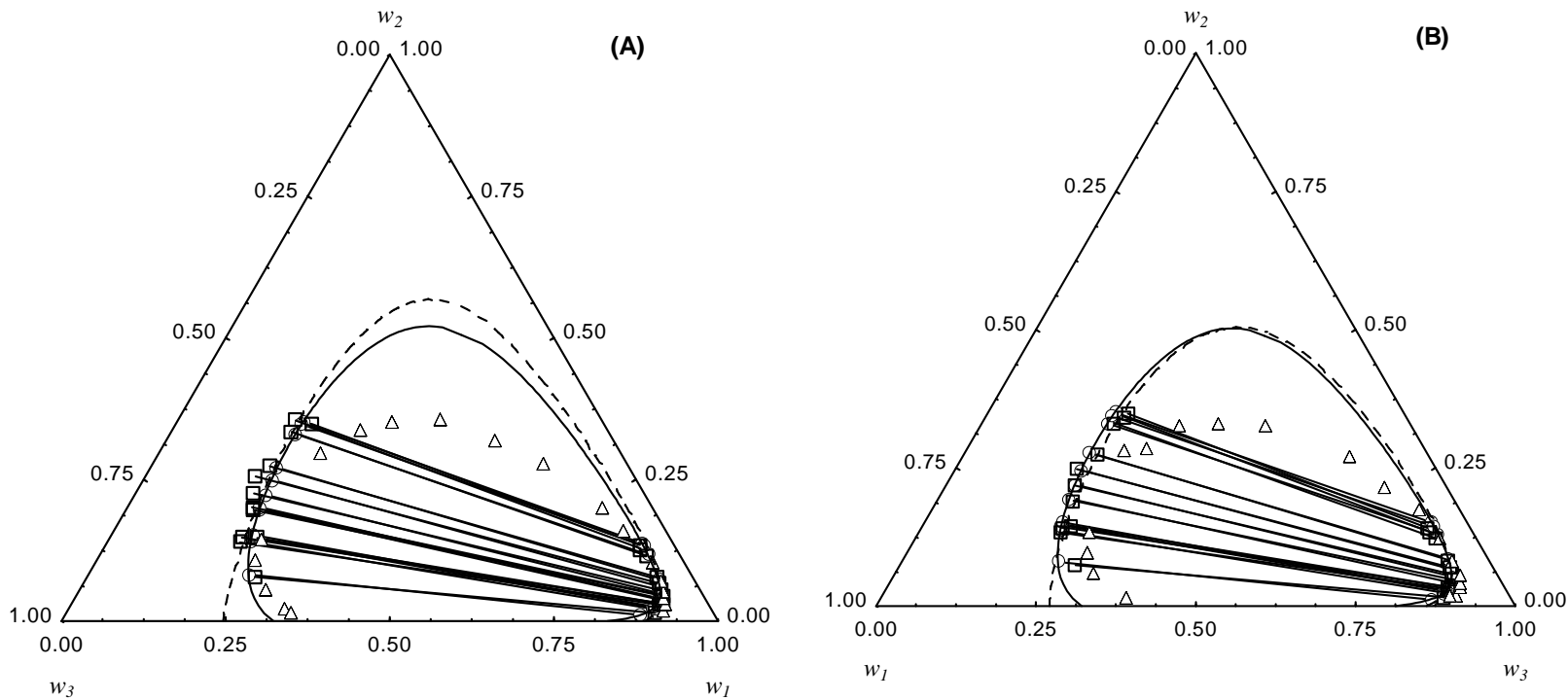

Figure 5 - Comparison of experimental ( $\odot$, binodal data by Guttman and Wilczura 1997, $\square-$ tie lines by Mafra and Krähenbühl 2006) and calculated values using UNIQUAC model ( - - , this work; -...-. parameters from Mafra and Krähenbühl 2006) for water(1) + acetone(2) + phenol(3) ternary system (A) at $323.15 \mathrm{~K}$ and (B) $333.15 \mathrm{~K}$ (in mass fraction).

Figure 6(A) presents the LLE phase envelope for the system water + acetone + phenol using the NRTL model with $\alpha_{\mathrm{ij}}$ parameter fitted (Set 2, Table 2). It can be observed that the model predicted the island occurrence for this system at temperatures higher than 393.15 K. Same behavior was observed considering the NRTL model with fixed values of $\alpha_{\mathrm{ij}}$ in the parameter correlation (Fig. 6B). From the binary system phenol-water was completely miscible at 340.15 and the results presented in Figure 6(A)-(B), it was observed that the NRTL model was capable of predicting the 
island formation qualitatively. Additionally, Figure 6(C) presented the LLE diagram for this system estimated from the application of UNIQUAC model at higher temperature conditions. It was observed that the UNIQUAC model provided similar results of that NRTL; however, the island formation occurred higher at $413.15 \mathrm{~K}$.
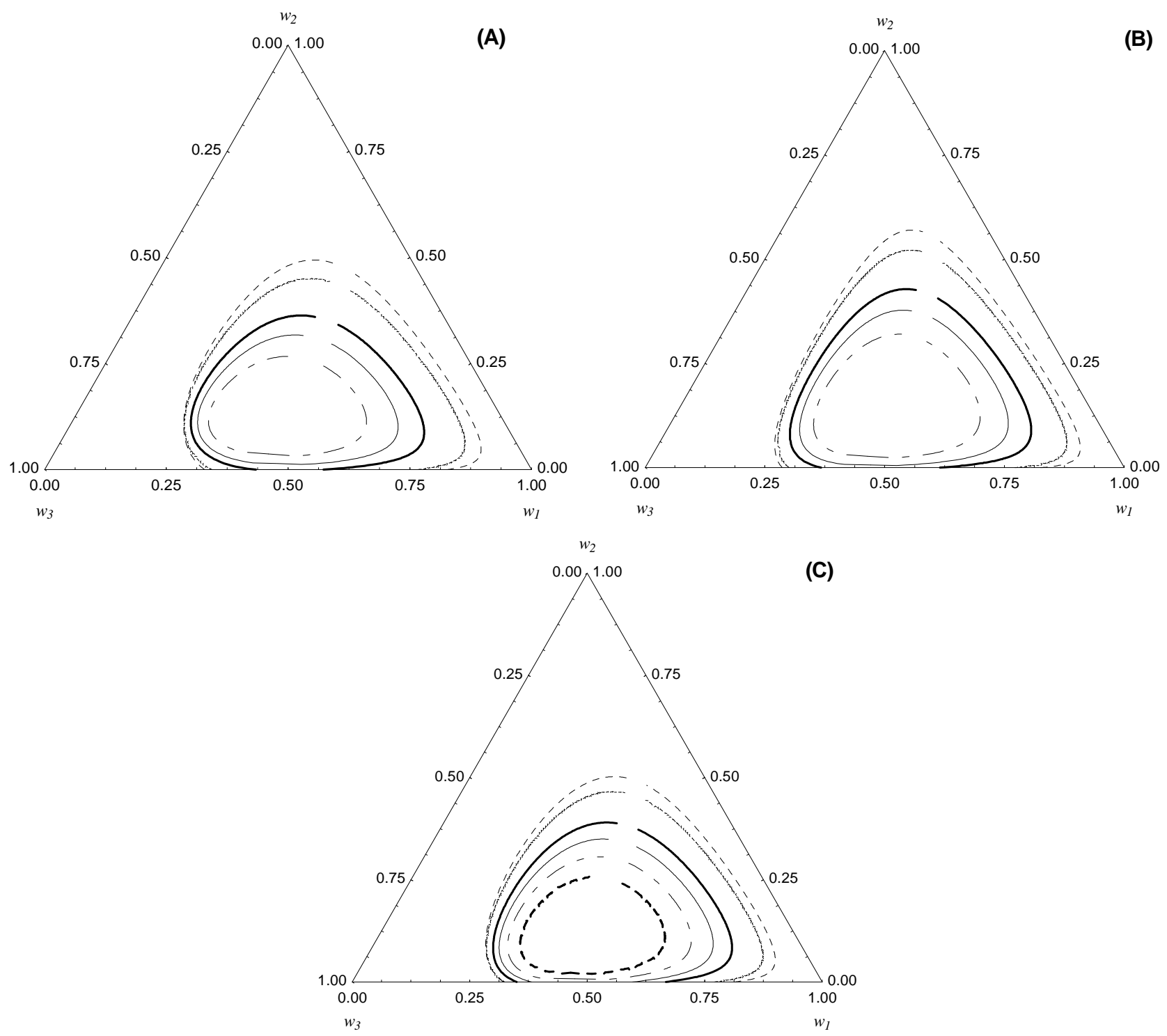

Figure 6 - Calculated values of LLE for water(1) + acetone(2) + phenol(3) system at (---.-, 333.15 $\mathrm{K} ; \cdots \cdots \cdots, 353.15 \mathrm{~K} ;-\frac{1}{2}, 393.15 \mathrm{~K} ;-, 413.15 \mathrm{~K} ;-\cdots, 433.15 \mathrm{~K} ;--\bullet, 453.15$ K), (A) NRTL model using aij fitted, (B) NRTL model using fixed aij and UNIQUAC model, (in mass fractions).

\section{CONCLUSIONS}

In this work, two sets of experimental data from the literature, one with the formation of island and other presenting a tendency to immiscibility island formation, were studied. In both the cases, the method proved to be efficient, not only in the stochastic correlation of the NRTL model, but also in the UNIQUAC correlation to the experimental data. It was observed that the systematic thermodynamic modeling of the liquid-liquid equilibrium data using NRTL model was even valid when the stochastic optimization method was used. It must be remembered that in the stochastic 
algorithm, there was no need for initial estimation of the parameters. However, the application of this relationship for the UNIQUAC model was not found for all the systems investigated. Therefore, it seemed that the use of a stochastic algorithm, coupled with the stability test, showed good potential as an important tool in phase equilibrium data modeling of complex systems, such as liquidliquid equilibrium with immiscibility island formation. Fundamentals of thermodynamic intermolecular pair potential energy were successfully employed to improve the interpretation of correlation results and present information for model parameters found from the experimental data fitting.

\section{ACKNOWLEDGMENTS}

The authors thank the CNPq, CAPES and MEC/REUNI for financial support and scholarships.

\section{REFERENCES}

Abrams DS, Prausnitz JM. Statistical thermodynamics of liquid mixtures: A new expression for the excess Gibbs energy of partly or completely miscible systems. AIChE J. 1975; 21: 116-128.

Corazza FC, Oliveira JV, Corazza ML. Application of a subdivision algorithm for solving nonlinear algebraic systems. Acta Sci Technol. 2008; 30: 27-38.

Ferrari JC, Nagatani G, Corazza FC, Oliveira JV, Corazza ML. Application of stochastic algorithms for parameter estimation in the liquid-liquid equilibrium modeling. Fluid Phase Equilibr. 2009; 280: 110-119.

Gmehling J, Jiding L, Schiller M. (1993), A modified UNIFAC model 2. Present parameter matrix and results for different thermodynamic properties. Ind Eng Chem Res. 1993; 32: 178-193.

Guttman TK, Wilczura H. Liquid-liquid equilibria for phenol-water-acetone system. Przem Chem. 1997; 76: 21-22.
Mafra MR, Krähenbühl MA. Liquid-liquid equilibrium of (water+acetone) with cumene or $\alpha$-methylstyrene or phenol at temperatures of ( 323.15 and $333.15 \mathrm{~K}) . J$ Chem Eng Data 2006; 51: 753-536.

Magnussen T, Rasmussen P, Fredenslund A. UNIFAC parameter table for prediction of liquid-liquid equilibria. Ind Eng Chem Proc Des Dev. 1981; 20: 331-339.

Michelsen M.L. The isothermal flash problem. Part II. Phase-split calculation. Fluid Phase Equilibr. 1980; 9: 21-40.

Olaya MM, Reyes-Labarta JA, Velasco R, Ibarra I, Marcilla A. (2008), Modeling liquid-liquid equilibria for island type ternary system. Fluid Phase Equilibr. 2008; 265: 184-191.

Prausnitz JM, Lichtenthaler RN, Azevedo EG. Molecular Thermodynamics of Fluid-Phase Equilibria. Third Edition. New Jersey. Prentice Hall PTR; 1999.

Press WH, Teukolsky SA, Vetterling WT, Flannery BP. Numerical Recipes in FORTRAN. Second Edition. Cambridge University Press; 1992.

Renon H, Prausnitz JM. Local compositions in thermodynamic excess functions for liquid mixtures. AIChE J. 1968; 14: 135-144.

Sorensen JM, Art W. Liquid-liquid Equilibria Data Collection, Chemistry Data Series, Parts 2, and 4, vol. 4. DECHEMA. Frankfurt; 1980.

Sorensen JM, Magnussen T, Rasmussen P, Fredenslund A. Liquid-liquid equilibrium data: Their retrieval, correlation and prediction Part I: Retrieval. Fluid Phase Equilibr. 1979a; 2: 297-309.

Sorensen JM, Magnussen T, Rasmussen P, Fredenslund A. Liquid-liquid equilibrium data: Their retrieval, correlation and prediction Part II: Correlation. Fluid Phase Equilibr. 1979b; 3: 47-82.

Sorensen JM, Magnussen T, Rasmussen P, Fredenslund A. Liquid-liquid equilibrium data: Their retrieval, correlation and prediction Part III: Prediction. Fluid Phase Equilibr. 1980; 4: 151-163.

Stragevitch L, d'Ávila SG. (1997), Application of a generalized maximum likelihood method in the reduction of multicomponent liquid-liquid equilibrium data. Braz J Chem Eng. 197; 14: 41-52.

Treybal RE. Liquid extraction. $2^{\text {th }}$ ed. New York: McGraw-Hill; 1963. 